\title{
ChARACTERIZATION OF EPITAXIAL LAYERS USING SCANNING MiCROWAVE MicrosCOPY
}

\author{
Tomas Martinek, Josef Kudelka, Milan Navratil, Vojtech Kresalek
}

Tomas Bata University in Zlin, Nad Stranemi 4511, Zlin 76005, Czech Republic

\begin{abstract}
In this article, atomic force microscopy and its variation scanning microwave microscopy were used for characterization of the epitaxial layers with different dopant doping levels specified by the manufacturing data. The vapour phase epitaxy was used for the deposition of individual layers on a silicon substrate. The measured data were visualized and compared with the manufacturing data.
\end{abstract}

Keywords: Epitaxial layers; dopant density; atomic force microscopy; scanning microwave microscopy; vapour phase epitaxy
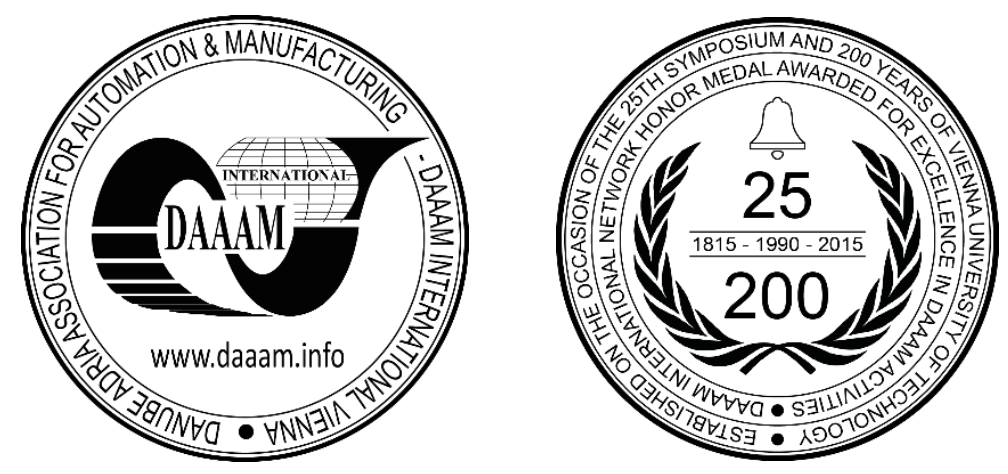

This Publication has to be referred as: Martinek, T[omas]; Kudelka, J[osef]; Navratil, M[ilan] \& Kresalek, V[ojtech] (2016). Characterization of Epitaxial Layers Using Scanning Microwave Microscopy, Proceedings of the 26th DAAAM International Symposium, pp.1109-1114, B. Katalinic (Ed.), Published by DAAAM International, ISBN 978-3-90273407-5, ISSN 1726-9679, Vienna, Austria

DOI: $10.2507 / 26$ th.daaam.proceedings. 156 


\section{Introduction}

Nowadays, with the development of electronics and microelectronics the complexity of electronic devices increased approximately four times every three years. For this reason, leading world producers invest into the development of electronic structures and networks in nano-scale. In this area, ultra-thin layers, nanocomposites and nanoparticles, among others, play an important role.

Ultra-thin layers are very interesting area of physics, with properties located between microphysical structures, where classic physics is used, and nanophysical structures, where quantum physics takes place. These ultra-thin layers have great potential in the industry, e.g. as diffusion barrier in electrical components fabrication [1]. These layers are also commonly used in semiconductor industry. Moreover, these layers can be used in photonics [2], optoelectronics [3] and spintronics [4]. Nanoparticles can be used for modification of the mechanical properties of materials [5] and nanocomposites, like ultra-thin layers, can be used to enhance the electrical properties of materials [6].

Measuring methods and capabilities for the quality control and diagnostics of these structures are significantly limited due to their structure size which is often approaching atomic resolution. There are only few possible measuring methods - diffraction techniques and microscopy - scanning electron microscopy and scanning probe microscopy. [7]

In the present work, we studied epitaxial layers with different dopant doping levels deposited on a silicon substrate by the vapour phase epitaxy $[8,9,10,11]$. Scanning probe microscopy techniques - Atomic force microscopy [12] and scanning microwave microscopy were used for the diagnostics of dopant doping changes among the growth layers, to confirm the manufacturing process. This was essential for following experiments. Scanning microwave microscopy is a derived method of atomic force microscopy that combines electromagnetic measurement capabilities of vector network analyzer with nanometer resolution of atomic force microscopy. [13] This allows simultaneous measuring of both topographical and electromagnetic properties of analyzed sample, which is a great advantage comparing to other measuring techniques.

\section{Materials and methods}

Several epitaxial layers with different dopant doping levels were deposited on a silicon substrate by the vapour phase epitaxy. The levels of doping were specified by the manufacturing data (Fig. 1.) from lowest dopant concentration approximately $\mathrm{C}=10^{16} \mathrm{~cm}^{-3}$ in depth of $1 \mu \mathrm{m}$ up to depth of $10 \mu \mathrm{m}$ with dopant concentration approximately $\mathrm{C}=10^{19} \mathrm{~cm}^{3}$.

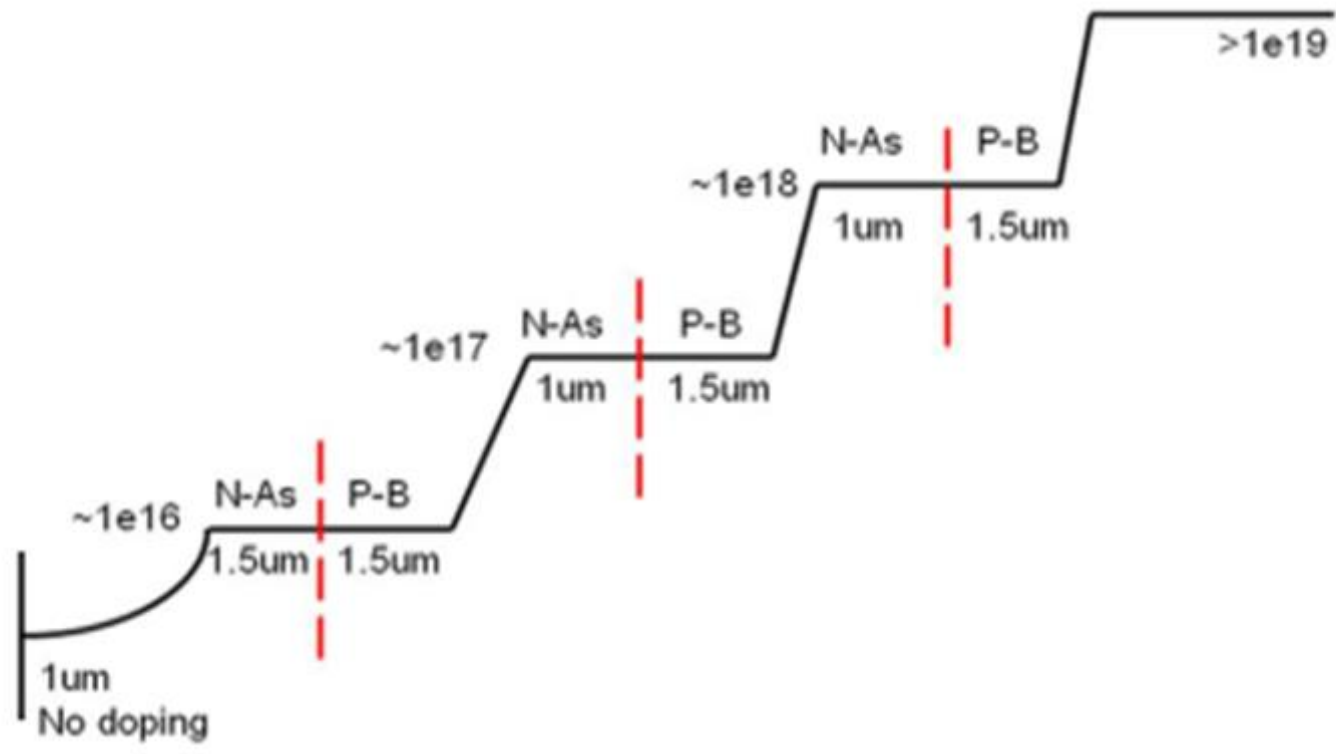

Fig. 1. Manufacturing data of the sample.

Scanning microwave microscopy and atomic force microscopy were used for characterization of the manufactured sample.

Atomic force microscopy is based on bending of cantilever by interatomic forces between atoms at the tip of the scanning cantilever and surface atoms of measured sample. These forces are attractive or repulsive depending on the distance between the tip and the sample. Bending of the cantilever due to tip-sample force is measured by laser beam which is reflecting from the upper part of the cantilever to four-segment photodiode. Voltage changes in each segment determine position of the tip. 


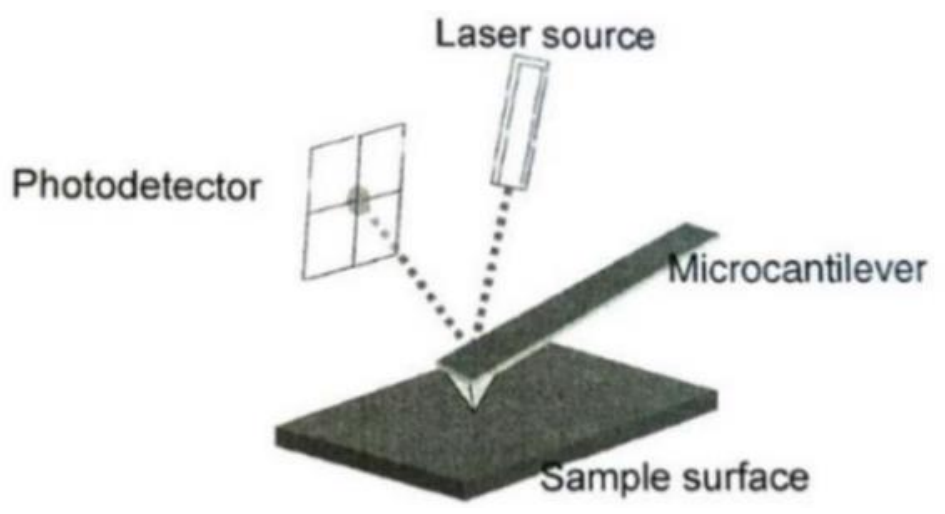

Fig. 2. Atomic force microscopy detection principle [14].

Scanning microwave microscopy can be easily understood using optical analogy. As shown in Fig. 3., when light strikes an object, some of the light is reflected from the object and some passes through depending on optical properties of the object. By measuring amount of light reflected and/or transmitted, optical properties of the object can be determined. Applying the same principle for a microwave spectrum, measurement of the incident, reflected and/or transmitted waves can provide detailed information about the properties of the material. [15, 16, 17]

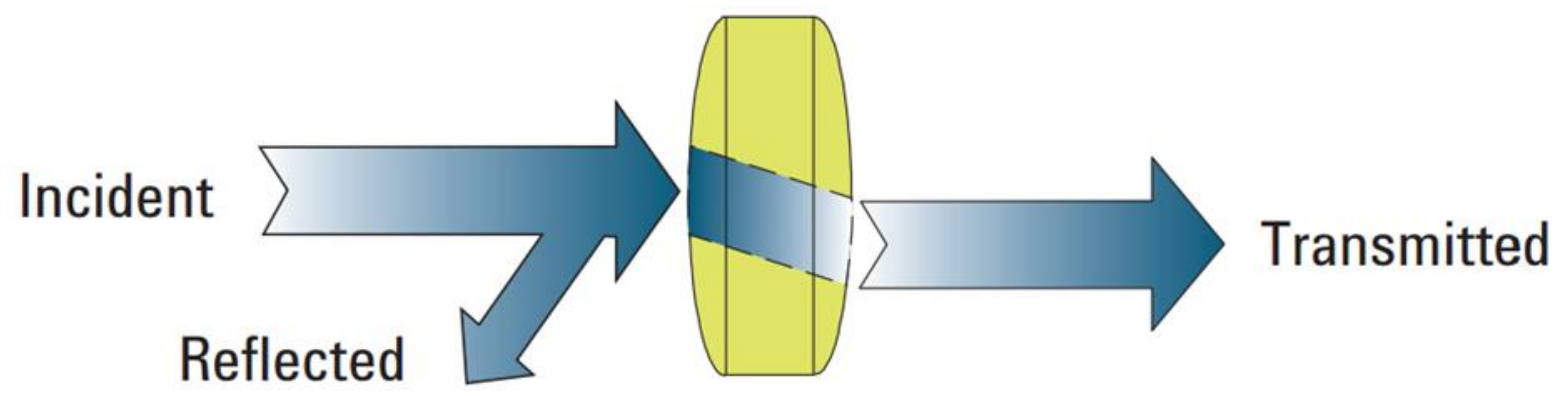

Fig. 3. Optical analogy to high-frequency characterization [16].

To measure the properties of the sample and its individual layers, the cross section of the sample was needed. The cut was performed using diamond pen. No other special preparation was necessary and all measurements were taken under common laboratory conditions.

The atomic force microscope Agilent 5420 SPM/AFM, vector network analyzer PNA N5230A and metal-coated (Pt-Ir) conductive AFM probe were used - size $400 \times 60 \mu \mathrm{m}$, spring constant of $0.3 \mathrm{~N} \cdot \mathrm{m}-1$, diameter less than $7 \mathrm{~nm}$, resonant frequency of $4.5 \mathrm{kHz}$. The vector network analyzer provided the microwave signal of frequency $2.468808 \mathrm{GHz}$.

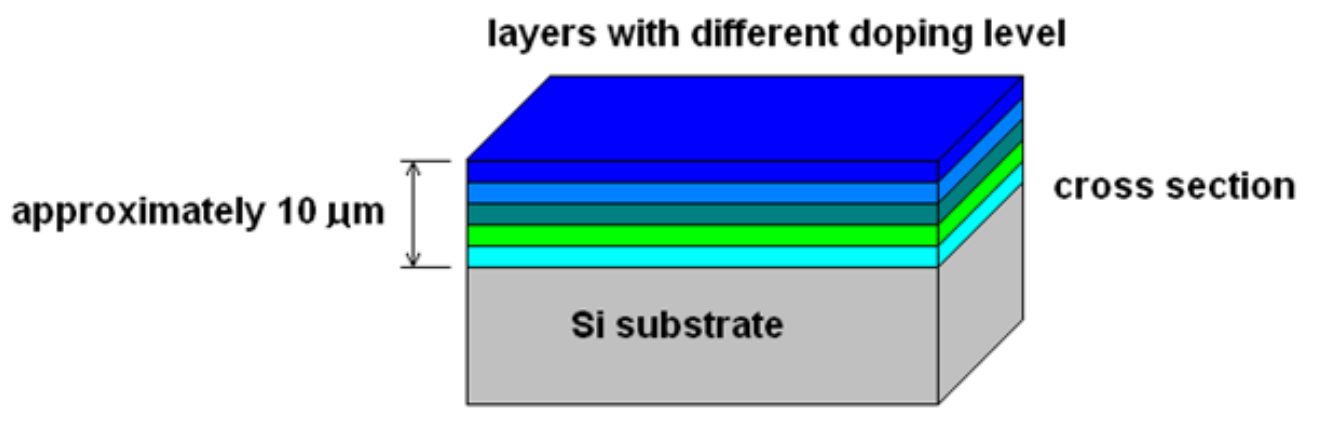

Fig. 4. Manufactured sample.

\section{Results and discussion}

Scanning area of $15 \times 15 \mu \mathrm{m}$ was chosen next to the upper edge of the manufactured sample to measure all growth layers at the cross section (Fig. 4.). AFM topography, PNA amplitude and dC/dV signal were measured, visualized and analyzed using Gwyddion [18] and MatLab software. Figure 5. shows topography of the measured sample. Topography was almost flat as expected. 


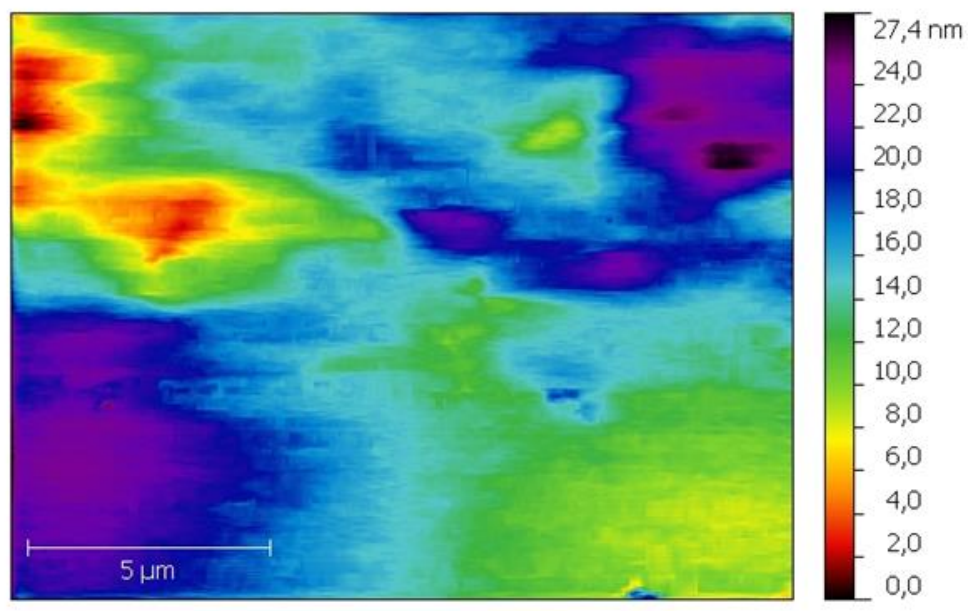

Fig. 5. Topography image.

The PNA amplitude has not provided any relevant information. However, the most useful results provided dC/dV signal as can be seen in Fig. 6., epitaxial layers with different doping concentration are visible in depths corresponding to manufacturing data.

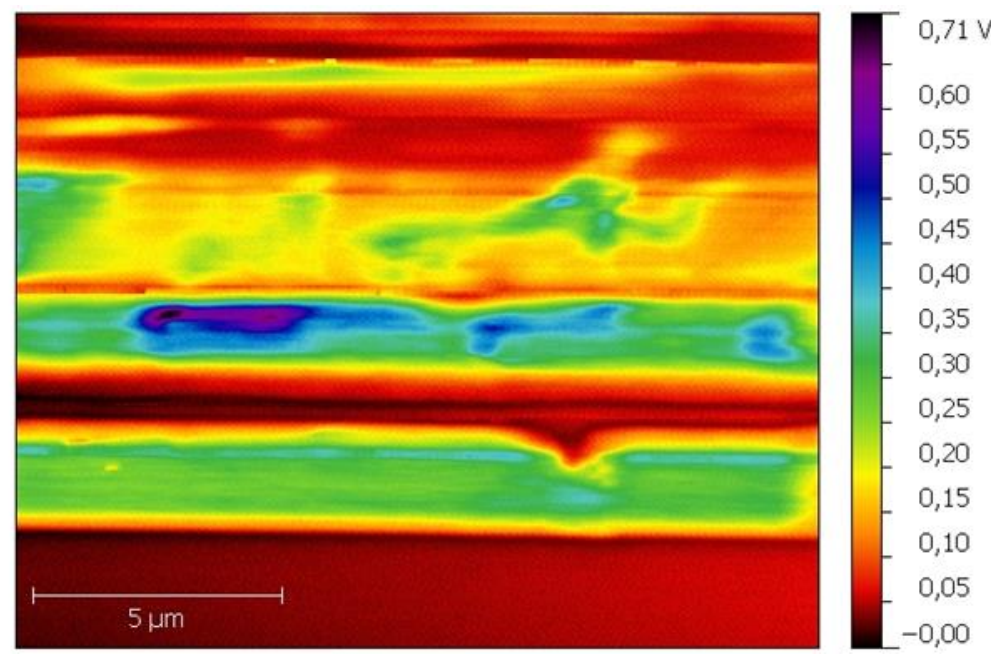

Fig. 6. dC/dV signal.

Figure 7. shows the extracted profile of the measured data. The horizontal axis represents a depth of the sample while the vertical axis represents $\mathrm{dC} / \mathrm{dV}$ signal.

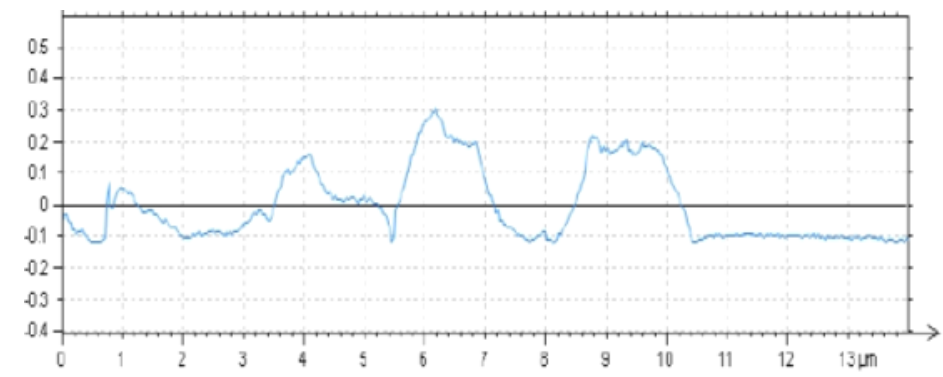

Fig. 7. Extracted profile from Fig. 6.

For the purpose of the comparison with the manufacturing data, the obtained data were approximated and integrated in MatLab software. Figure 8. presents the integrated profile of the measured signal. The vertical axis is relative. Calibration standard would be needed to get absolute values of the dopant concentration; however, when compared with 
the manufacturing data (Fig. 1.), it is obvious that both figures correspond and each concentration is in the correct depth of the sample.

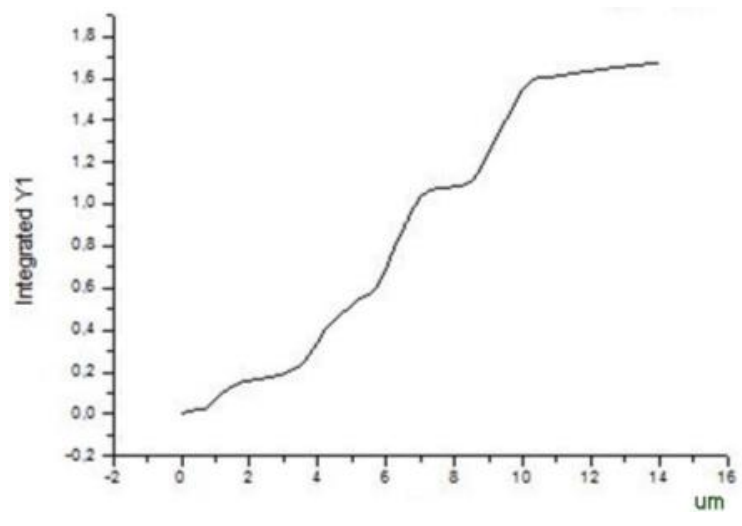

Fig. 8. Integrated profile extracted from Fig. 6.

The experiment has shown that scanning microwave microscopy can be powerful tool to probe electromagnetic properties on scales of micro or nanometer. The informative character obtained by scanning microwave microscopy reveals it is one of the most suitable techniques for the quality control in semiconductor industry and for control of the ultra-thin layers and nanostructures. A limitation of the study was approximation of the data to calculate the integration. Secondly, the results are only relative. To get absolute values calibration standard would be needed. In the future research, the measured sample will be modified using nanolithography.

\section{Conclusion}

Submicron characterization of several epitaxial layers with different dopant doping concentration deposited on a silicon substrate by the vapour phase epitaxy was presented using atomic force microscopy and scanning microwave microscopy. Scanning microwave microscopy showed the potential for submicron characterization of epitaxial layers with different properties and for the control of a deposition processes.

\section{Acknowledgements}

This work was performed with financial support by the Ministry of Education, Youth and Sports of the Czech Republic within the National Sustainability Programme project No. LO1303 (MSMT-7778/2014) and with support by Internal Grant Agency of Tomas Bata University under project IGA/CebiaTech/2015/045 and IGA/FAI/2015/048.

\section{References}

[1] I. Fernandez, X. Borris and F. Pérez-Murano, Atomic force microscopy local oxidation of silicon nitride thin films for mask fabrication, Nanotechnology, Vol. 16, No. 11, pp 2731-2737, 2005.

[2] H. Yonezu, Y. Furukawa and A. Wakahara, III-V epitaxy on Si for photonics applications, Journal of Crystal Growth, vol. 310, Issue 23, pp 4757-4762, 2008.

[3] T. Pauporte, D. Lincot, Electrodeposition of semiconductors for optoelectronic devices: results of zinc oxide, Electrochimica Acta, vol. 45, Issue 20, pp. 3345-3353, ISSN 0013-4686, 2000.

[4] M. Akabori, V. A. Guzenko, T. Schäpers and H. Hardtdegen, InxGa1-xAs/InP selective area metal-organic vapor phase epitaxy for non-magnetic semiconductor spintronics, Journal of Crystal Growth, vol. 310, Issue 23, pp 4821 4825, 2008.

[5] Z. Pesina, J. Sopousek and J. Bursik, Mechanical properties of joints created by silver nanoparticles, Annals of DAAAM for 2012 and Proceedings of the 23rd International DAAAM Symposium, ISBN 978-3-901509-91-9, ISSN 2304-1382, pp 0139 - 01421, Vienna, Austria, 2012.

[6] M. Murarescu, D. Dima, G. Andrei and A. Circiumaru, Influence of Mwcnt dispersion on electric properties of nanocomposites with polyester matrix, Annals of DAAAM for 2011 and Proceedings of the 22nd International DAAAM Symposium, ISBN 978-3-901509-83-4, ISSN 1726-9679, pp 0925-0926, Vienna, Austria, 2011

[7] P. Hawkes and J. C. Spence, Science of microscopy, New York: Springer, c2007, ISBN 03-872-5296-7

[8] G. Du, Y. Ma, Y. Zhang and T. Yang, Preparation of intrinsic and N-doped p -type ZnO thin films by metalorganic vapor phase epitaxy, Applied Physical Letters, Vol. 87, Issue 21, 2005, article number 213103, pp 1-3, DOI: $10.1063 / 1.2132528$.

[9] A. Tsukazaki et al, Repeated temperature modulation epitaxy for p-type doping and light-emitting diode based $\mathrm{ZnO}$, Nature Materials, Vol. 4, Issue 1, 2005, pp. 42-45, DOI: 10.1038/nmat1248. 
[10] E. García-Tabarés and I. Rey-Stolle, Impact of metal-organic vapor phase epitaxy environment on silicon bulk lifetime for III-V-on-Si multijunction solar cells, Solar Energy Materials and Solar Cells, Volume 124, 2014, Pages 17-23, ISSN 0927-0248, http://dx.doi.org/10.1016/j.solmat.2014.01.034.

[11] D. Salomon et al, Metal organic vapour-phase epitaxy growth of GaN wires on Si (111) for light-emitting diode applications, Nanoscale Research Letters, Volume 8, Issue 1, 2013, Pages 1-5, ISSN 1931-7573, DOI: 10.1186/1556-276X-8-61

[12] G. Binnig, C. F. Quate and Ch. Gerber, Atomic force microscopy, Physical Review Letters, Vol. 56, Issue 9, 1986, pp 930-933, DOI: 10.1103/PhysRevLett.56.930.

[13] W. Hen, Introduction to scanning microwave microscopy, Agilent Technologies, USA, 2008, 5989-8881EN, Available online: https://www.agilent.com/cs/library/applications/AN-IntroSMM_5989-8881.pdf.

[14] V. V. Tsukruk and S. Singamaneni, Scanning probe microscopy of soft matter: fundamentals and practices, Wiley, ISBN 978-3-527-32743-0, 2011.

[15] Undestanding the fundamental principles of vector network analyzer, Agilent Technologies, USA, 2012, 59657707EN, Available online: http://cp.literature.agilent.com/litweb/pdf/5965-7707E.pdf

[16] S. Wu and T. Hopson, SMM imaging of dopant structures of semiconductor devices, Application Note, Agilent Technologies, USA, 2014, 5991-0562EN, Available online: http://cp.literature.agilent.com/litweb/pdf/59910562EN.pdf

[17] H. P. Huber et al, Calibrated nanoscale dopant profiling using scanning microwave microscope, Journal of Applied Physics, vol. 111, Issue 1, art. no. 014301, DOI: 10.1063/1.3672445, 2012

[18] D. Necas and P. Klapetek. Gwyddion: an open-source software for SPM data analysis, Central European Journal of Physics, vol. 10, No. 1, pp. 181-188, 2012. 